\title{
Smart Health Care System-An Easy First Aid Solution
}

\author{
V. Vijayashangavi \\ Faculty of Computing \\ Sri Lanka Institute of \\ Information Technology \\ Colombo, Sri Lanka
}

\author{
M. M. A. Ibrahim \\ Faculty of Computing \\ Sri Lanka Institute of \\ Information Technology \\ Colombo, Sri Lanka
}

\author{
U. M. Azaad \\ Faculty of Computing \\ Sri Lanka Institute of \\ Information Technology \\ Colombo, Sri Lanka
}

\author{
F. M. U. Farook \\ Faculty of Computing \\ Sri Lanka Institute of Information Technology, \\ Colombo, Sri Lanka
}

\author{
S. G. S. Fernando \\ Senior Lecturer \\ Faculty of Computing \\ Sri Lanka Institute of Information Technology, \\ Colombo, Sri Lanka
}

\begin{abstract}
First Aid matters a lot in emergency situations which may even decide between life and death. Utilizing modern technology to encourage use of the First aid at vital situations is the area of interest of this Smart Health Care System. Numerous accidental and injurious death have occurred in the past which could have been avoided by giving emergency first Aid treatments. Lack of first aid knowledge, unavailability of first aid medication, passing responsibilities into next hands and ultimately ending up with no eligible first aider taking responsibility have been identified as the main reasons for failed emergency cases without First Aid. Currently there are first aid teams which provide first-aid services. Waiting till the expertise to arrive to the spot worsens the situation. Smart Health Care System (SHCS) (Easy First Aid Solution) is a perfect medicine vending mechanism which has been designed to cope with the above issues. SHCS mainly consist of a kiosk network which act as an interactive first aid vender which dispense suitable medication according to the emergency situation. These Kiosks also provides assistance to do first aid treatment by giving effective instruction. Hence, Smart Health Care Kiosk act as both vending purpose kiosk and information kiosk. This combined idea gives uniqueness to SHCS. Furthermore SHCS mobile Application has been developed to give online ordering facility by which just entering unique code will be enough to make purchase. Time spent at kiosk has been planned to be reduced with this feature. This app also displays nearby kiosk with available medication. This increase the efficiency of the system. Central administration has been implemented to maintain reliable control over kiosk network. Overall functionalities in SHC System harnesses efficiency of providing first-aid and ultimately benefits success rate of medical emergency cases.
\end{abstract}

\section{Keywords}

First- Aid, KIOSK network, Website, Android Application, Embedded System

\section{INTRODUCTION}

First aid is an immediate help given to a sick or an injured person before a professional medical treatment, in order to avoid worsening health conditions. This immediate aid can make a huge change in a victim between life and death. According to the statistics 150000 of people die unnecessarily due to lack of first aid [1]. While survey carried out by St. John Ambulance found that $24 \%$ of people were feeling reluctant to give first aid to struggling victims whereas $59 \%$ of people were not feeling confident in trying to save a life [2]. Being this the current situation, as researchers there arises a vital need to encourage people towards first aid practices. There are lots of organizations around the world which emphasize on first aid as their main vision. These organizations carryout first aid training programs and provide pre-hospital ambulance services to the affected victims. Simply the knowledge of first aid does not help at all the situation without enough resources, i.e. medical kits. Therefore a technology which could assist even a norm to become a first aider by providing necessary knowledge and resources is a crucial need. In Sri Lanka road traffic kills average one person in every 4.5 hours [3]. Whereas $33 \%$ of these deaths could have been avoided if necessary first aid has been supplied [3]. From the studies discussed above it can be found that public is so much reluctant to be first aiders but go behind pre-hospital ambulance services and hospitals in first place which is not a best practice in critical situations. Therefore, an attractive user friendly technology which could train or instruct people then and there to provide first aid using necessary first aid kit has been identified as the most reliable solution to cope up with these issues.

Smart Health Care System is an all in one system which contains kiosk network which provides necessary first-aid kits and First-aid knowledge, mobile application which provides efficient and effective user services such as online ordering, expiry date notification and other support services and finally a website as a backend which controls overall system processes. A Patient or a first-aider will be able to purchase any first-aid kit at any time using SHC kiosk. Furthermore, relevant instruction to use any first aid kit will also be provided through SHC kiosk as well as SHC mobile application. RFID payment method have been implemented to make the medicine purchase much faster. First-aider will also be able to collect the online orders just by entering the reference number (Order number) to the kiosk. Past order details, purchase details and balance details can also be retrieved through SHC mobile application. Whenever firstaider or patient wishes to get notification on expiry date of any medicine he can simply make that request by scanning barcode of that medicine through his/her mobile application. Although limited stock can be maintained in a kiosk, SHC System has ensured availability of stock all the time by practicing a stock management algorithm. This algorithm anticipates the demand of medicine and monitors the stock level of kiosks all the time. If any kiosk stock level is found less in number compared to demand stock level, it notifies through admin website. Backend website allows admin to monitor these stock levels, sales details, order details, and other related information. Admin will also be able to generate 
reports regarding sales and kiosk stock. Overall objective achieved through SHC System is the increased effectiveness of purchasing and using first aid kits and basic pharmaceutical medicines with new Internet of Things based technologies.

Remainder of the research paper are structured as follows. Different literature papers studied and respective advantages and disadvantages of each researches have been explained in section 2 of this paper. Section 3 briefly describes the processes carried out at each phase of system development lifecycle. All the results of this extensive research have been discussed in section 4 followed by conclusion and future works in section 5 .

\section{BACKGROUND STUDY}

The literature review is done based on several components related to this proposed system so that the proposed system could be identified and differentiated among the other related products in the market. The study is based on the following topics which is GPS technology, GSM Modules, Arduino Boards and technology, Android application and also about similar existing systems.

Two books called "Vital First Aid"[4] and "What should be in your First Aid Kit"[5] were studied to identify the methods of first aid techniques which could be used to help a victim in medical emergencies and various types of first aid kits that can be used

When the Software in Automated vending Machines are considered Mr. FRODE et al. have discussed the User Interface designs for public kiosks referring to design heuristics and mistakes in public kiosks. It's based on the mistakes in the Taiwan high Speed Rail Ticket Vending Machine particularly [6]. And Mr. Peter et al. has invented a Vending machine with synthesized description messages. It's a vending machine adapted to reproduce audio and video messages relating to the products being dispensed [7].

When Mobile Application related to Health care is considered Errol et al. [8] have proposed a system to provide a comprehensive and up-to-date summary of the role of the smartphone in medicine by highlighting the ways in which it can enhance continuing medical education, patient care, and communication and the use of these apps by students while on clinical rotations can also support education at the bedside. This could translate into an improved quality of education that could be a focus of examination.

Borja et al. [9] have researched on identifying the lack of apps which are most required at the moment in the medical field and emphasizes to increase the amount of it to treat the eight most prevalent health conditions at the moment.

Gugapriya et al. [10] have proposed a system for mobile banking applications to locate the nearest ATM center by using the Global Positioning System (GPS) It helps in the navigation to the nearest ATM centre.

When General Packet Radio Service Applications are considered Mahesh et al. [11] has proposed a system regarding GSM (Global System Messaging) based secured device control system using App Inventor for Android mobile phones. By this system home appliances could be operated via automation system with GSM facility.

ShingFong [12] has proposed a system that allows a vending machine to accept coins. The selected beverage will be dispensed when user has inserted sufficient amount of cash. The balance will be returned to the user after the beverage has

\section{been delivered.}

Raj and Naveen [13] have proposed a system to identify fake currency detection system using raspberry pi which identifies the denomination and counterfeit of currency. The approach uses multiple ways of image processing to do the job.

To identify Separate distribution techniques in Kiosks the following studies were made. Thomas et al. Have invented a dispenser in order to reduce theft in shops using a product pushing device to do the dispensing. The main intention is to avoid Theft of small items in retail stores which is a common problem [14].

Thomas et al. have invented a vending machine with flexible plastics. This helps products to be always available in front in a shelf once the product in front is taken out [15].

The study of above research papers tells us that there's no specific Kiosk that's capable of doing First-aid vending which is locatable and accessible through mobiles. Researches prove that presently the vending machines in the market function as product dispensers only (dispenses products when payment is done). Another main drawback is the lack of "First-aid Knowledge" among people. Even though people have access to medicines at home or elsewhere the proper way of use is unknown.

Injuries and health issues are unavoidable but the risk of it can be reduced through proper first-aid.

The SHC kiosk is capable of providing First-Aid medicines, Give out instructions/guides of doing first-Aid and get the payments. It comes along with a mobile app which is capable of locating the kiosk \& facilitating the online ordering providing the instructions/guides in the mobile app itself.

\section{METHODOLOGY}

This section discusses about all the tasks carried out at each phase of System Development Lifecycle in detail together with respective deliverables produced at each phase

\subsection{Planning}

Current problems faced by the society regarding efficient first-aid treatments during emergency situation was thoroughly studied. Set of functionalities were chose as solutions and initial planning for the development of Smart Health care System was carried out. As a part of this planning process, feasibility analysis was carried out to determine the feasibility of this system for public and medical authorities in economical, technical and organizational context. Then, Prototype methodology was chose as development methodology of Smart Health Care System because the project team was new to some of the technologies used and scope was complex. Subsequently, A Work Breakdown Structure (WBS), Gantt chart and Budget for initially identified functionalities were prepared.

\subsection{Requirement Gathering and Analysis}

Issuing online questionnaires and reviewing past researches published on similar topics were carried out as part of requirement gathering process. Questionnaires were issued to the sample size of 33 people selected from the full population based on Non-Probability Quota sampling techniques. Based on the analysis of questionnaire results project team found that living environment has influence in availability and accessibility of first aid kits. Furthermore, it was found that most people deny being a first-aider at emergency situations due to fear and lack of knowledge. Lack of proper stock management was also found as another factor which affects 
the efficiency of giving first-aid. Based on the above analysis of primary data basic functional requirements of Smart Health care system was finalised. They are providing 24/7 first-aid vending through kiosk, providing interactive vending pattern to give first-aid knowledge effectively and maintaining proper backend to maintain stock at kiosks according to the requirements to ensure availability of first-aid kits at all emergency times.

Additionally literature review was carried out on similar systems available and systems with technologies which were chose to build Smart Health Care System. By analyzing those research papers drawbacks and limitations were found out. Major research gap found and solutions to those gaps in Smart Healthcare System was finalized. Details about this study has been provided under section 2 of this research paper.

\subsection{Designing}

High level architecture diagram was sketched as the first process in the designing phase in order to determine overall operational model of Smart Health Care System. Next, Use case diagrams and use case scenarios were finalised and Entity relationship diagrams were drawn accordingly. Soon after that, hardware interface diagram and interfaces relevant for Smart Healthcare System mobile application and backend website were designed in parallel. Designing suitable algorithm for efficient stock management in kiosk was another important process which was carried out at this phase. Finally, Decisions on development platforms for hardware, mobile application, website, and database servers were finalised.

Fig 1 shows the High Level Architecture diagram of Smart Healthcare System sketched at this phase.

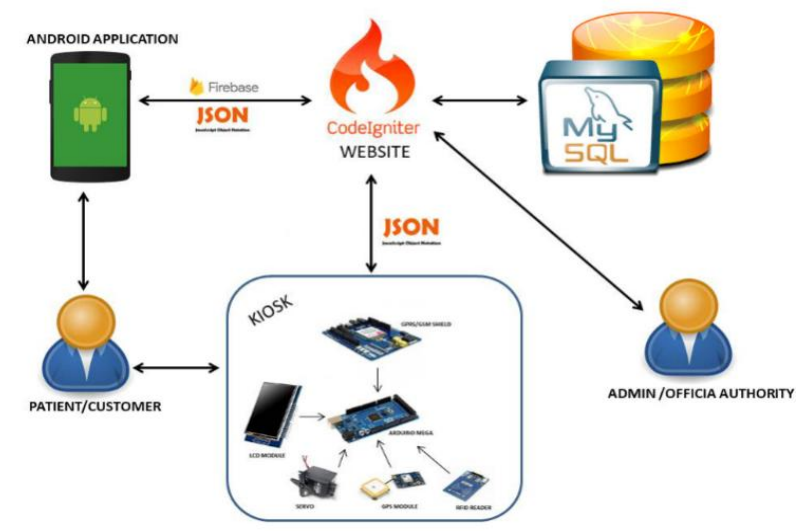

Fig 1: High level diagram

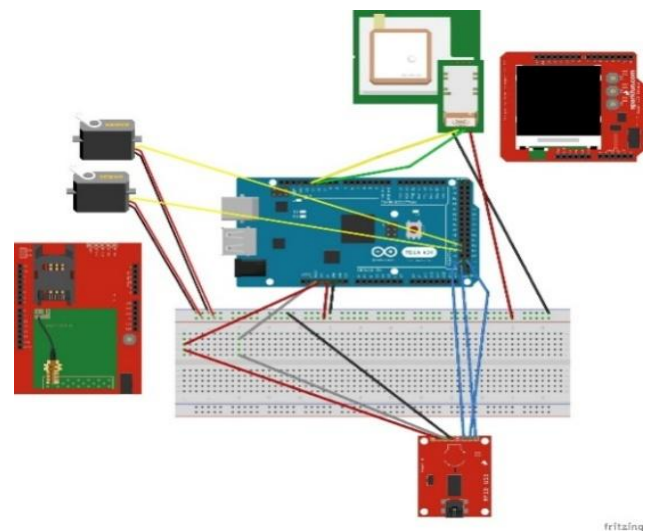

Fig 2: Hardware infrastructure diagram
Fig 2 depicts the Hardware infrastructure diagram of Smart Healthcare System sketched at this phase.

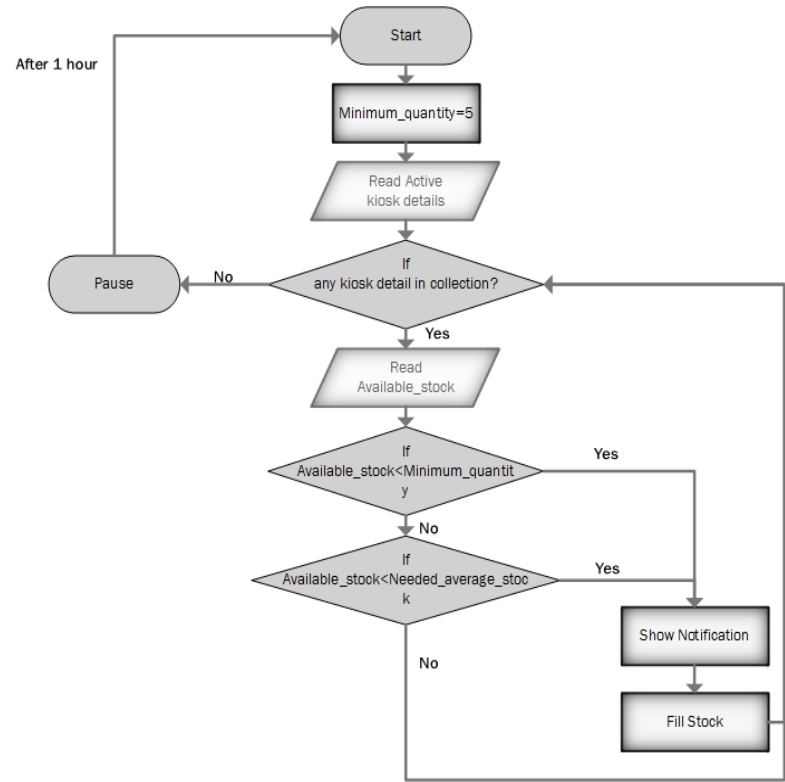

Fig 3: Stock management algorithm flow chart

Fig 3 depicts the Flow Chart for Stock Management Algorithm designed at this phase.

\subsection{Implementation}

Actual Smart Health Care System was developed according to the designs produced at designing phase. Both hardware and software components of Smart Healthcare System were developed in parallel. All the hardware components were connected to Arduino MEGA 2560 and relevant instruction for Servo motor, TFT LCD Display, RFID Reader and GSM/GPRS Shield were coded and installed using Arduino IDE. Initially whole vending process was developed to work independently without connecting to common database. Meanwhile, Android mobile application was developed using Android Studio and Website was developed using Code Igniter PHP framework, Bootstrap framework and JQuery framework in Brackets and PHPStrom. Initially, website was hosted locally in WAMP server and MySQL database in phpMyAdmin was used for both android application and website. Soon after the Development of individual components, website and database were hosted and relevant PHP pages were also coded for integration with hardware components. Firebase Cloud Messaging services was used to send expiry date push notifications to android application. Google map android API and Google map JavaScript API was used to display and pin relevant markers to relevant kiosk locations. Overall, HTML, CSS, PHP, JAVA, Micro C and JavaScript were used for the development of Smart Healthcare System.

\subsection{Testing}

Unit Testing, Integration testing, System testing and Performance testing were carried out ensure reliability of entire Smart Healthcare System. Soon after the development of individual components were finished unit testing was carried out by using both black box and white box testing. Then Hardware components were integrated one after another and integrated testing was carried out. At the same time, mobile application and website components are also integrated together and tested. Finally, system testing was carried out after integrating hardware and all software 
components. Timing tests, security and concurrency tests were carried out for order processing and stock management to ensure reliable performance of the system.

\section{RESULTS AND DISCUSSION}

Section 4 discusses the results and their discussion that the research team achieved from the research project. The important implications of the research findings, regardless of the statistical significance of this research are discussed below. Further, Identifying the defect and limitation of this project can be useful for future researchers in order to continue their research. The major object of "Smart Health Care System" is to provide first aid medicines and kits at any time using online ordering system and from kiosk itself.

Smart Health Care System comprises of three major components such as Website Application, Android Application for Domestic users and admins, and a Kiosk application

Primarily, the Kiosk is designed to read the user input both the online order and manual order and to dispense the first aid kits. Location of kiosk was also read and transmitted to the main server. Fig 4. below shows design and hardware implementation of the Smart Kiosk.

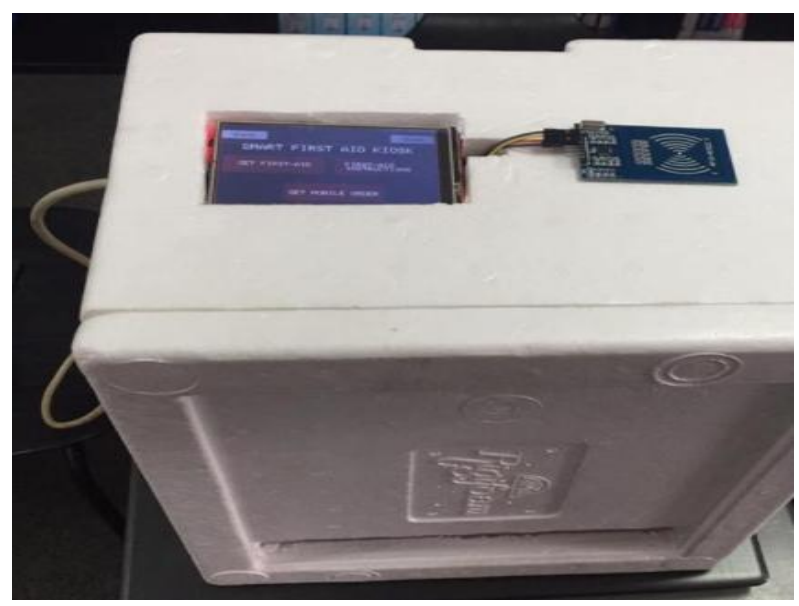

Fig 4: Kiosk design

The kiosk related details received from kiosk and stored in the main website application which is handled by the admin for proper stock management. Fig 5. below shows the website application interface which shows the kiosk stock details.

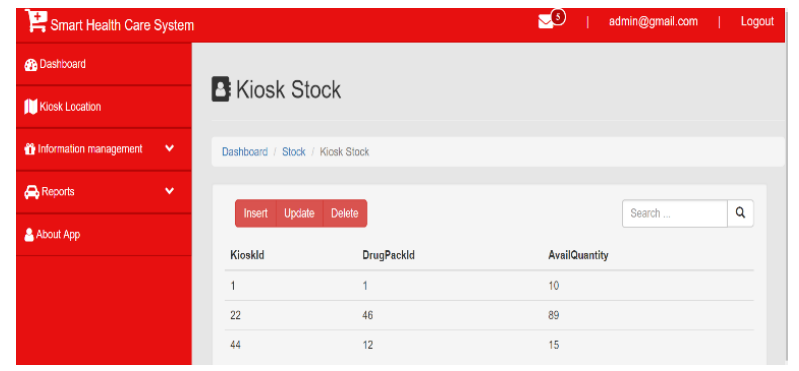

Fig 5: Kiosk management interface of backend website

Initially, customers can register themselves by using the Android mobile application to use the online ordering system.

Next, the customers details will be send to the main server and the admin can view the customer's details and he has the right to keep them or to remove them. Fig 6 . below shows the Android mobile application interface relevant to this process

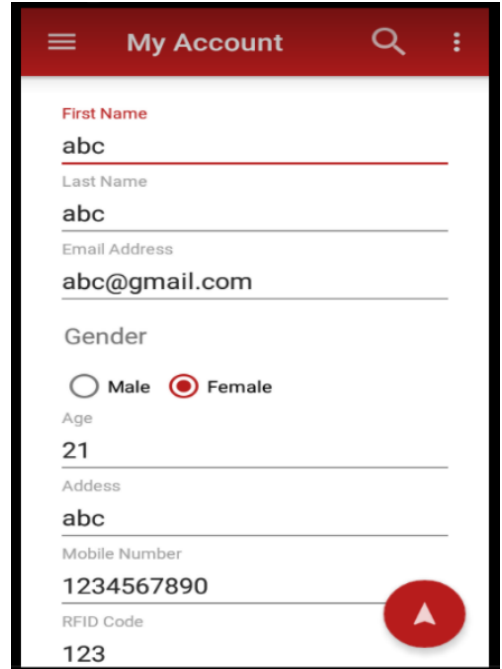

Fig 6: My account mobile interface

After registering in to the system customers will be able to check the stock and the kiosk where the stocks available using the mobile application. Fig 7. below shows the interface where available locations of selected first aid kit is shown with respective available stock in each kiosk.

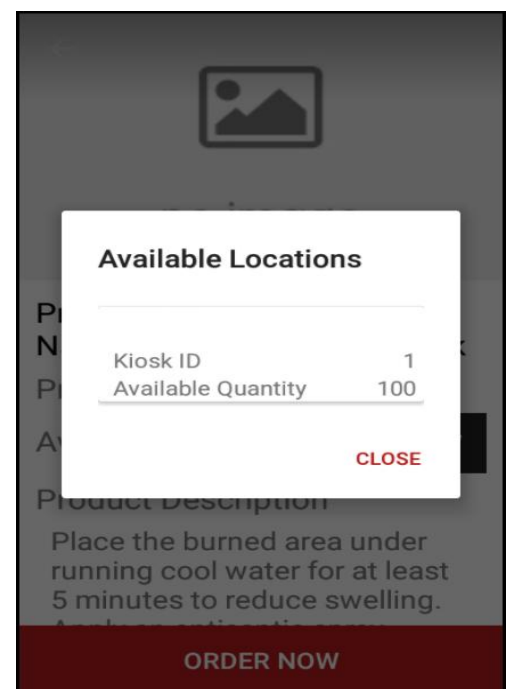

Fig 7: Available location mobile interface

Soon after the customers selects needed first-aid kit, it will be shown in the "My Orders" interface. Following Fig 8. shows the interface where the ordered items are listed

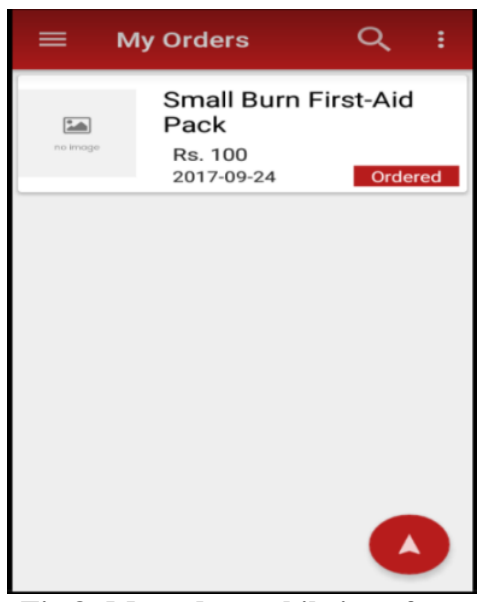

Fig 8: My order mobile interface 
Fig 9. Below "My Balance" interface which shows the outstanding amount available in the users' Rfid card.

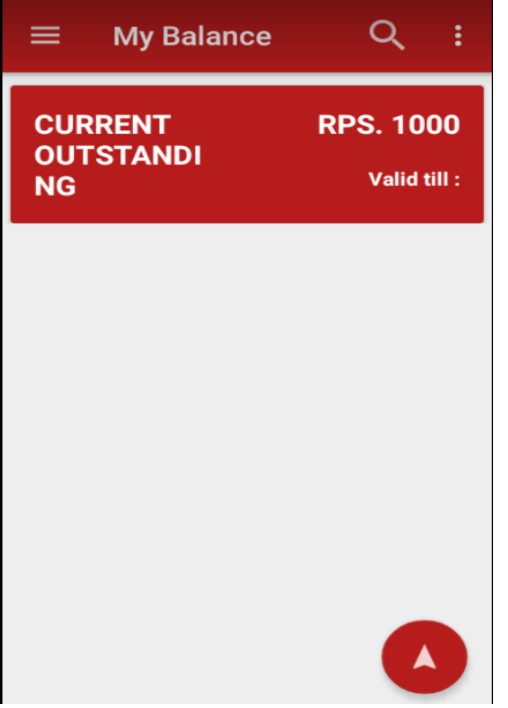

Fig 9: My balance mobile interface

Real-time monitoring about kiosk stock is achieved through Google map API embedded in the main website application. Whereas kiosk locations and its stock status are shown. Fig 10. below shows the main website application interface used for monitoring purpose and location maps displayed to the Admin.

Soon after each and every stock details are updated to the main website application. Reports regarding the transaction and sales are generated by the main website application. Fig 11. and Fig 12. below shows the sales report and Transaction reports generated by the system.

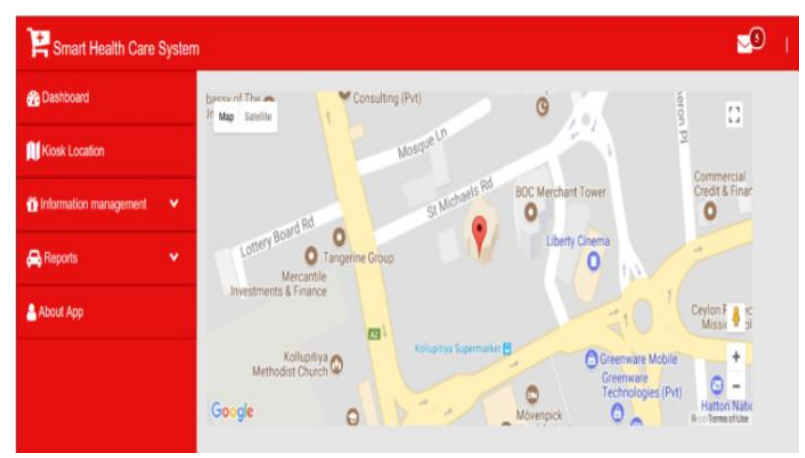

Fig 10: Real time monitoring backend interface

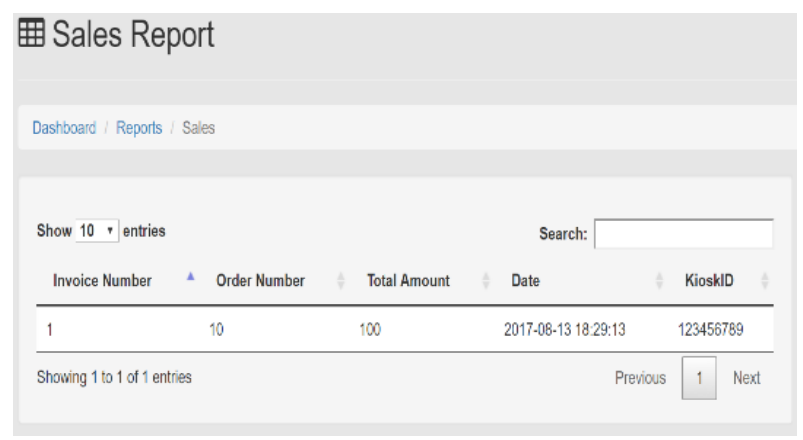

Fig 11: Sales report - backend interface

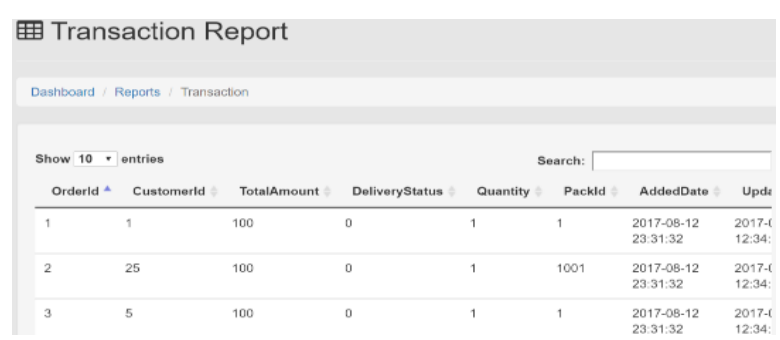

Fig 12: Transaction report-backend interface

Reliability and accuracy of the system are crucial factors which need to be considered in medical related applications. Moreover Smart health Care System is a dedicated system for efficient first aid giving purposes where reliability and accuracy are most important. Based on various tests carried out in various environments and conditions, Smart Health Care System has showed 95\% reliability and 79\% accuracy.

Fault tolerance, recoverability, authentication and trustworthiness are main reliability factors which were considered when testing reliability of the system. After series of testing and error correction finally reliability of the system has been achieved by the following functionalities. Reliability of the system on peak times has been achieved by the use of Stock Management Algorithm which automatically calculates and compares needed amount of medical stock and available stock of each and every kiosk and alerts the backend admin to fill out the stock. Kiosk failures and breakdowns are immediately figured out by the system by monitoring the time interval in which the real time information are received by the backend from each and every kiosk. If any malicious is observed it is also alerted to the admin. Furthermore, a quick service check during every stock filling is also suggested to discover any breakdowns. User Authentication is ensured by using safe login system in mobile application and backend website. Moreover, Expiry date notification functionality of the system ensures that none of the system users will consume expired medicines. Accuracy level was calculated by testing hardware devices and results of the stock management algorithm. Most testing gave successful result expect for the GPS Module and TFT LCD Display. GPS Module reads no values unless it has connectivity to at least three satellites. So during rainy and cloudy seasons it failed the accuracy tests. On the other hand due to the use of simple research purpose TFT display with less sensitivity, it failed some initial tests and additional TFT libraries were used to rectify these errors logically

\section{CONCLUSION}

For any developing country, health care sector plays an important role. It's important that every student in their curriculum are been taught about first aid that has to be provided at critical situations. The main objective of developing a smart health care system based kiosk is to provide an efficient automated system which allows vending of first-Aid kits effectively. This system would develop a platform to increase the usage of first aid in Sri Lanka which could facilitate benefits to the all kind of users.

This system is expected to be launched near commercial areas, beaches, and highly populated areas with high health risks. Smart health care system provides instructions in a user friendly manner as this would eventually increase the usage and knowledge of first aids kits. This system would help the doctors since initial first aid would help them to treat the patients immediately. 
During the development of this project, the following limitations were identified:

- $\quad$ Need of data plan enabled SIM.

GSM/GPRS uses mobile sim data to transfer real time data about Kiosks' status to the server. Therefore, data plan should be enabled in this case and recharging is need to be maintained for uninterrupted connection with the central server.

\section{- $\quad$ Limited battery life.}

A $12 \mathrm{v}$ 8000mah li-ion battery has been used to power up Arduino circuit. It has a battery life of circular charge and discharge up to 500 times. Battery should be recharged in regular intervals for reliable functioning of Smart Kiosk.

- Less accuracy with GPS location readings.

Location of kiosks have been read using GPS module which need at least 3 satellite connections to accurately read a location. Hence location read may be with less accuracy in case of less satellite connections.

- RFID tag and Basic knowledge is significant for Domestic user to manipulate the kiosk.

This system mainly considers about first aid treatments and health care benefits by such system but still large scope with regards to this systems are open for future research. Till the goal has been met, a numerous number of research paths would be open to the researchers. This research area will stay fresh with the rise of modern technologies. The project team has identified future scope of this project which may interest to the researchers in this area. They are as follows,

- Based on the data collected about the kiosk sales, data mining can be applied to get more knowledge about health risks in a particular area.

- Medicinal usage alerting system can be built to ensure correct usage of medicines.

- Based on the medicines purchased relevant authorities like ambulance and police can be notified automatically.

- Doctor prescriptions can be uploaded to relevant patients account as a mobile order which could be purchased at any kiosks.

- Kiosks can be further developed to identify health conditions and prescribe medicine or suggest relevant medical checkup according to the user given symptoms.

\section{ACKNOWLEDGMENTS}

We would like to declare our honest sense of gratitude to our institution - Sri Lanka Institute of Information Technology (SLIIT). We are acutely beholden to whose help, stimulating suggestions, knowledge, experience, and courage helped us in all the times of study and analysis of the project in the pre and post research period. Also very special thanks to Dr. Yasas Jayaweera for their endless support given at times of difficulty as well as to our seniors and the lecture panel. The completion of this undertaking could not have been possible without the participation and assistance of so many people whose names may not all be enumerated.

\section{REFERENCES}

[1] "Lack Of First Aid Skills Endangers Up To 150,000 Lives". The Guardian. N.P., 2017. Web. 22 Mar. 2017.

[2] "Two Out Of Three People Couldn't Save A Life". www.sja.org.uk. N.P., 2017. Web. 22 Mar. 2017.

[3] "Trauma Secretariat - Sri Lanka Ministry Of Healthcare and Nutrition". Traumaseclanka.gov.lk. N.P., 2017. Web. 22 Mar. 2017.

[4] V. Renn and N. Humphries, Vital first aid, 1st ed. [Camberwell, Vic.]: Penguin, 2010.

[5] H. Sawyer, What Should be in your First Aid Kit, 1st ed. Bedford, 2011.

[6] SANDNES, FRODE EIKA et al. User Interface Design For Public Kiosks: An Evaluation Of The Taiwan High Speed Rail Ticket Vending Machine. 1st ed. Taiwan: JOURNAL OF INFORMATION SCIENCE AND ENGINEERING, 2010. Web. 22 Mar. 2017.

[7] S. Vogel, Peter. "Vending Machine With Synthesized Description Messages". 1992: n. pag. Print.

[8] Errol Ozdalga, Ark Ozdalga, Neera Ahuja, " The Smartphone in Medicine: A Review of Current and Potential Use Among Physicians and Students", [Online]. Available: http://www.jmir.org/2012/5/e128/pdf. [Accessed: 19mar- 2017].

[9] Borja Martínez-Pérez, Isabel de la Torre-Díez, Miguel López-Coronado, "Mobile Health Applications for the Most Prevalent Conditions by the World Health Organization", JOURNAL OF MEDICAL INTERNET RESEARCH, vol.15,issue 6,2013

[10] GUHAPRIYA A,VAITHEKI J and KAVIYARASI S, "Mobile Banking With Location Tracking Of Nearest ATM Center Using GPS". Gugapriya A* et al. / (IJITR) INTERNATIONAL JOURNAL OF INNOVATIVE TECHNOLOGY AND RESEARCH Volume No. 1.Issue No. 3 (2017): 253 - 255.

[11] Mahesh N. Jivani," GSM Based Home Automation System Using App-Inventor For Android Mobile Phone", 1st ed. International Journal of Advanced Research in Electrical, Electronics and Instrumentation Engineering, Vol. 3, Issue 9, September 2014.

[12] TEOH SHING FONG, "IMPLEMENTATION OF BEVERAGE VENDING MACHINE USING FPGA". International Journal of Engineering Science and Innovative Technology (IJESIT) Volume 3.Issue 4 (2014).

[13] Swami Gururaj and Naveen,"Identification Of Counterfeit Currency And Denomination Using Raspberry Pi". International Journal of Innovative Research in Electrical, Electronics, Instrumentation and Control Engineering Vol. 4.Special Issue 2 (2016).

[14] Lang, Thomas et al. "Dispensing System". 2010: n. pag. Print.

[15] Thomas, A. Lockwood, and K. Miller Roger. "Product Dispensing System". 2014: n. pag. Print 